\title{
E1 movimiento anti-vacunas y la anti-ciencia como amenaza para la Salud Pública
}

\author{
The anti-vaccine movement and the \\ anti-science as threat for public health
}

Julián-Alfredo Fernández-Niño ${ }^{1}$; Hernando Baquero-L ${ }^{1}$

Forma de citar: Fernández-Niño JA, Baquero H. El movimiento anti-vacunas y la anti-ciencia como amenaza para la Salud Pública. Rev Univ Ind Santander Salud. 2019; 51(2): 103-106. doi: http://dx.doi.org/10.18273/revsal.v51n2-2019002 @) (1)

El movimiento anti-vacunas no es sino una consecuencia más del anti-intelectualismo, y del subsecuente fortalecimiento de la seudociencia, incluso en los países más desarrollados. La corriente anti-intelectual, y dentro de ella, la anti-ciencia, es muy antigua. Los filósofos naturales, los ilustrados del renacimiento, los evolucionistas o más recientemente, los científicos que estudian el cambio climático han tenido que enfrentarse históricamente, a hostigamientos, desprestigios y ataques sin bases lógicas, provenientes de diversos movimientos sociales, incluyendo: la religión organizada, grupos políticos reaccionarios al progreso, e incluso, no pocas veces, emporios económicos, cuando los hallazgos o teorías científicas han comprometido sus agendas o intereses.

Sin embargo, los movimientos anti-científicos del siglo XXI son diferentes en al menos dos aspectos a sus predecesores. En primer lugar, sus motivaciones no siempre están alineadas con los intereses económicos o de control bio-político, $\mathrm{y}$, en segundo lugar, porque ahora el internet les permite que se expandan y organicen en redes acéfalas, donde pueden difundir y perpetuar sus ideas ${ }^{1}$; además sus creencias se nutren de noticias falsas o testimonios anecdóticos no verificables, que rápida y eficientemente pueden difundirse para continuamente estar reclutando o radicalizando seguidores. Lamentablemente, los científicos no siempre disponen del tiempo, las herramientas de convencimiento o el interés para deliberar con estos sistemas de creencias, por lo que terminan, como sano mecanismo de defensa, displicentemente ignorándolos con la no deseada consecuencia del fortalecimiento social de la anti-ciencia y la seudociencia en el discurso público.

De forma más general, el sentimiento anti-científico, la tecnofobia y la desconfianza en las instituciones, como determinantes de la seudociencia, obedecen también a las frustraciones colectivas por el limitado acceso a información o peor aún, por la escasa participación en la toma de decisiones del público general, derivadas a su vez de sus limitaciones para comprender el conocimiento técnico-científico disponible, pero sobre todo sus bases lógico-racionales. Lo anterior termina convirtiéndose en un terreno fértil para que las teorías conspirativas se vuelvan sucedáneos al conocimiento empírico científico, y terminen psicológicamente dignificando al supuesto excluido del conocimiento, al hacerle pensar que hay algo que él sabe que los demás no, y que es su deber informarlo a todos. Esto, por ejemplo, ha sido descrito recientemente en el caso de las vacunas como un efecto psicológico de Dunning-Kruger, donde aquellos más hostiles a ellas son justamente las que menos entienden sus fundamentos ${ }^{2}$. Es esperado entonces que estos sistemas seudocientíficos logren cautivar más fácilmente a las personas con menos competencias en pensamiento crítico, exponiéndonos peligrosamente al error simplista de catalogarlos a todos como ignorantes, cuando el problema es más complejo y estructural. Sí bien parece claro que existe un estilo cognitivo que favorece la creencia en teorías conspirativas, sus bases pueden comprometer también valores, ideologías, sentimientos, posiciones políticas, creencias religiosas y frustraciones colectivas, de modo que comprender las bases del éxito para la difusión de estos sistemas de creencias constituye por sí mismo un reto de investigación, siendo indispensable entenderlas primero para poderlas combatir ${ }^{3}$.

1. Universidad del Norte. Barranquilla. Colombia 
Las teorías conspirativas, como sistemas explicativos alternos, parecen ser exitosas socialmente porque proveen explicaciones para lo que no se comprende, o no quiere comprenderse racionalmente, reivindicando prejuicios y creencias que necesitan psicológicamente ser ratificados continuamente. Generalmente se trata de entramados complejos de complots, secretos, amenazas y riesgos, en los que las personas encuentran satisfacción al sentirse como iluminados, asumiendo incluso en muchos casos la misión de vida de hacer algo al respecto. La militancia activa entonces se vuelve común en estos movimientos. Parte del problema para la deliberación pública con las personas que defienden estas ideas, es que, al no tener sus afirmaciones una base lógico-racional, no siempre apreciarán a los argumentos racionales como contraparte. Mientras tanto pueden recurrir al miedo, los prejuicios y emociones para incrementar su penetración social, y su influencia política, con consecuencias diversas, y a veces impredecibles.

Las personas, por ejemplo, que, en el siglo XXI, aún creen que la tierra es plana pueden terminar siendo inofensivas aparentemente, pero los negacionistas del cambio climático y los de los movimientos anti-vacunas están colocando directamente en riesgo la Salud Pública (Figura 1); su peligro aumenta a medida que logran más seguidores pues terminan influyendo en decisiones individuales y políticas que nos afectan a todos. La opción de desestimar estos sistemas de creencias sin deliberar ante ellos ha sido un gran error; la falta de debate además de terminar legitimando en cierta medida la pseudociencia, le otorga la ventaja de no exponer sus contradicciones, sus fallas lógicas, la ausencia de evidencia de sus afirmaciones y, sobre todo, las consecuencias de dar sus afirmaciones como ciertas.

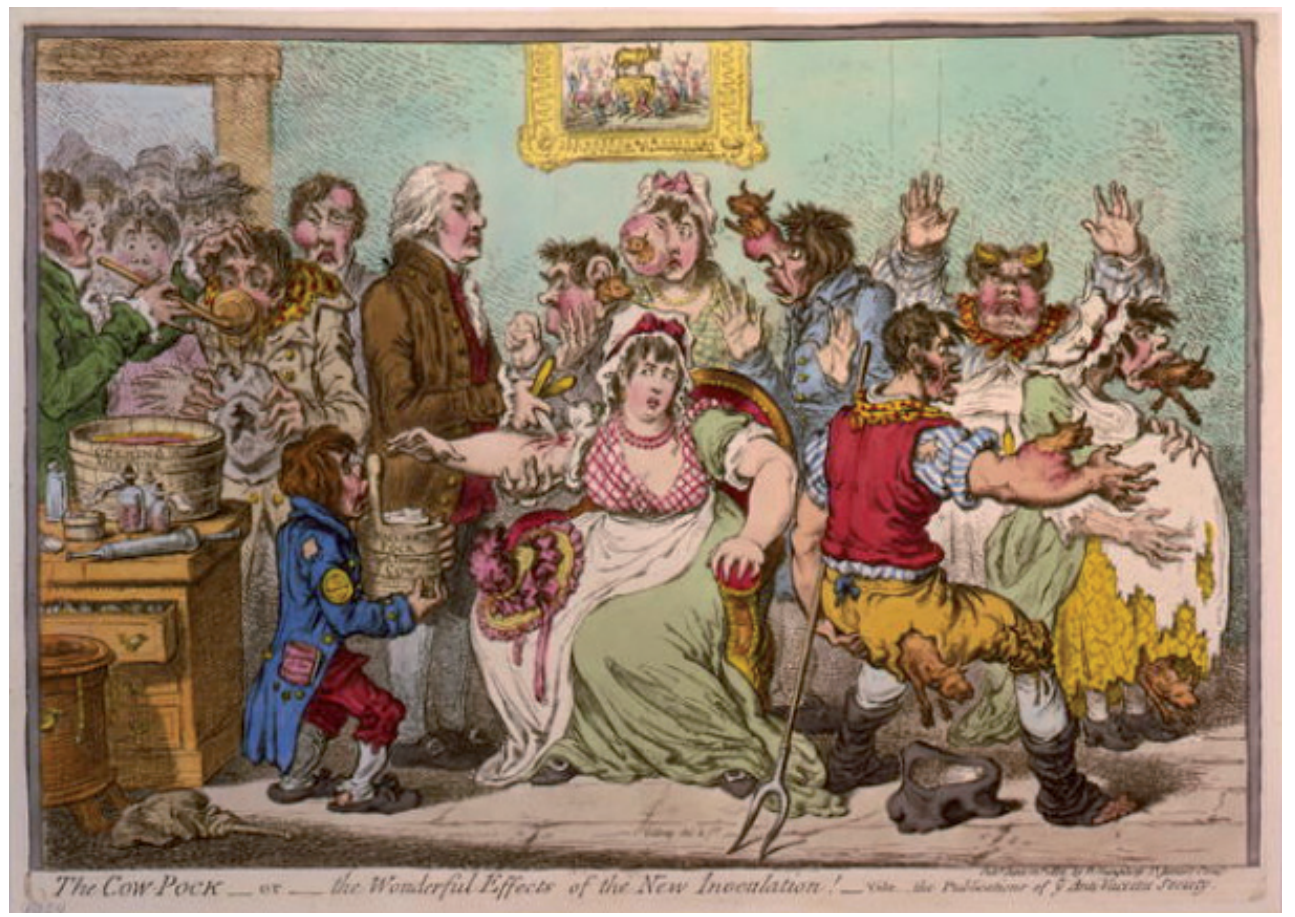

Figura 1. La picadura de vaca - o - los maravillosos efectos de la nueva inoculación. Caricatura de James Gillray (1756-1815). Sin restricciones conocidas para publicar. Tomado de: http://www.loc.gov/pictures/item/94509853/

Los problemas como el cambio climático, la evolución o la seguridad de las vacunas, no deberían ciertamente ser susceptibles a un debate de opinión. Se trata de hechos, y los debates políticos o filosóficos deberían ser sobre las ideas, incluso sobre los modelos que explican un conjunto de hechos o sus implicaciones éticas, políticas o filosóficas, pero no sobre los hechos per se. Hechos en el sentido que son tangibles, medibles y que permiten hacer predicciones precisas. Cuando se trata de hechos, estos ciertamente se pueden, y se deben cuestionar, por ello se contrastan y se ponen a prueba, a través de la replicación, pero no por la opinión, sino usando el método científico. De este modo, las personas y las civilizaciones, podrían tener miles de interpretaciones distintas del movimiento de los cuerpos estelares, pero sólo existe un sistema de conocimiento, basado en la observación, y la medición 
que puede predecir con exactitud un eclipse varias décadas antes. Todas las civilizaciones desde la antigüedad que predijeron eclipses usaron la ciencia. De este modo, para pesar de nuestros amigos posmodernos, y como lo puso en evidencia Alan Sokal ${ }^{4}$, la ciencia no puede ser considerada como otro sistema de creencias más. Esto no significa que la ciencia sea infalible, al contrario, la falibilidad, y su carácter revisionista son sus principales virtudes, pero es con evidencia empírica, y usando modelos explicativos coherentes derivados, como se establece que una vacuna es segura, no a través del debate de creencias. El dialogo de saberes es muy relevante para determinar los valores que debe tener una sociedad, para las decisiones políticas y para la democracia, pero no para establecer las realidades de la naturaleza.

En el caso particular de los movimientos anti-vacunas, y utilizamos el plural porque no todos tienen el mismo origen y los mismos fundamentos, se cumplen los criterios antes expuestos. Son sistemas de creencias generalmente acéfalos, con motivaciones variadas, y diversos grados de militancia que hacen esfuerzos colectivos para convencer a otros de no vacunarse ${ }^{5}$. Un factor común adicional es la desconfianza incurable en el conocimiento oficial, en el saber científico (que además no siempre es formalmente institucional), y cierta inclinación a aceptar teorías conspirativas con diverso grado de irrealidad (complots entre gobiernos y la industria farmacéutica, médicos guardando secretos de la cura de las enfermedades o su verdadera letalidad, hasta sistemas de control mental operados con las vacunas $)^{6}$. En sus fundamentos de creencias concurren varias vertientes, desde la quimiofobia, donde se promueve un estilo de vida anti-tecnológico, donde todas las creaciones humanas, particularmente aquellas mal catalogadas como "químicas" se consideran amenazas, encontrando acogida en estilos de vida "New Age" (categoría ambigua donde todo cabe), y nuevas reglas del cuerpo, y su relación con él ambiente, propias más de países occidentales desarrollados. En otros casos, el motor es el miedo, impulsado por la percepción de que fuerzas oscuras ocultan la verdad al público sobre lo inseguras que son las vacunas, y en ese caso encuentran coincidencias con las teorías conspirativas de los Estados. En esta militancia se invita a las personas a cuestionar lo que les han dicho, pero paradójicamente al tiempo que invitan a dudar, lo cual suena saludable, ya que la duda razonable es indispensable para el progreso del conocimiento, lo hacen en detrimento de la ciencia, o de la lógica misma, y a favor de fuentes de información a las que no juzgan con la misma fuerza, sin notar sus inconsistencias lógicas, su falta de evidencia, e incluso el nivel de absurdo al que pueden llegar?

Las vacunas son, junto a los antibióticos, una de las grandes conquistas de Salud Pública de la historia, y sin exagerar una de las mayores conquistas de la humanidad sobre la naturaleza. No cabe duda que gracias a ellas, hemos incremento la esperanza de vida, y, sobre todo, reducido la mortalidad infantil ${ }^{8}$. Desde su implementación han sido víctimas de campañas de desprestigio, sólo que ahora los movimientos anti-vacunas tienen un mayor alcance social. Podemos reconocer que en cierto punto es válido, una desconfianza racional hacia todo lo que es nuevo y desconocido, esa es la manera en que nos blindamos de los riesgos, el problema es cuando esto persiste contra todo el peso de la evidencia. Adicionalmente, ya no se trata de un problema solamente de lo que es o no verdadero científicamente, sino que tienen unas implicaciones directas en la vida de millones de personas. La Organización Mundial de Salud (OMS), ha estimado que el 30\% del incremento de brotes de sarampión a nivel mundial se deben al movimiento anti-vacunas ${ }^{9}$, y en todo el mundo se están viendo sus impactos. Una sola generación que en los países desarrollados no conoció el polio ni la viruela no debería tener el poder de regresarnos al pasado. No podemos entonces callar, tampoco reprimir de forma autoritaria estas creencias, pero los científicos definitivamente sí tienen que salir a deliberar ${ }^{7}$. No podemos seguir ignorando estos movimientos mientras se fortalecen más.

Sam Harris se pregunta constantemente en sus conferencias "¿Qué tipo de argumento lógico podemos ofrecerle a quién no aprecia la lógica? Esta es la cuestión, no es fácil dar un debate, cuando para comenzar los argumentos científicos no son apreciados por la contraparte. Sin embargo, como recomendó una guía de la $\mathrm{OMS}^{10}$, debemos pensar en que cuando deliberamos con anti-vacunas lo hacemos para que nos escuchen otros, hay personas, como los niños, en las que aún se puede despertar el pensamiento crítico, que todavía valoran el peso de los hechos, y que pueden cuestionarse aquellas cosas en que los han convencido. En este camino, debemos entender las bases psicológicas y socioculturales de la seudociencia si queremos combatirla, y estar dispuestos a sentarnos pedagógicamente a hablar con todos, porque algo de responsabilidad tenemos como científicos en la pérdida de confianza por el público, y si nuestro imperativo ético es la preservación de la vida, debemos bajarnos del altillo, y comenzar a hablar de las bases del pensamiento científico, una vez más y todas las veces que sea necesario. 


\section{Referencias}

1. Smith N, Graham T. Mapping the anti-vaccination movement on Facebook. Inf Commun Soc. 2017; 1(1): 1-18. doi: 10.1080/1369118X.2017.1418406.

2. Motta M, Callaghan T, Sylvester S. Knowing less butpresuming more: Dunning-Kruger effects and the endorsement of anti-vaccine policy attitudes. Soc Sci Med. 2018; 211: 274-281. doi: 10.1016/j.socscimed.2018.06.032.

3. Dagnall N, Drinkwater K, Parker A, Denovan A, Parton M. Conspiracy theory and cognitive style: A worldview. Front Psychol. 2015; 6(206). doi: 10.3389/fpsyg.2015.00206.

4. Alan Sokal JB. Impostura intelectuales. 1998. 68 p.

5. Orr D, Baram-Tsabari A. Science and politics in the polio vaccination debate on Facebook: A mixed-methods approach to public engagement in a science-based dialogue †. J Microbiol Biol Educ. 2018; 19(1): 1-8. doi: 10.1128/jmbe.v19i1.1500.

6. Hussain A, Ali S, Ahmed M, Hussain S. The Anti-vaccination movement: A regression in modern medicine. Cureus. 2018;10(7). doi: 10.7759/cureus.2919.

7. Wolfe RM. Anti-vaccinationists past and present. BMJ. 2002; 325(7361): 430-432. doi: 10.1136/ bmj.325.7361.430.

8. Greenwood B. The contribution of vaccination to global health: past, present and future. Philos Trans R Soc. 2014; 1796: 9. doi: 10.1098/rstb.2013.0433.

9. World Health Organization. Measles cases spike globally due to gaps in vaccination coverage. 2018. p. 3 .

10. World Health Organization (WHO). How to respond to vocal vaccine deniers in public. Best practice guidance. 2016. 